\title{
Associations of physical activity and screen time with white matter microstructure in children from the general population
}

\author{
María Rodriguez-Ayllon ${ }^{\text {a }}$, Ivonne P.M. Derks ${ }^{\text {b,c }}$, Michiel A. van den Dries ${ }^{\text {b,c }}$, \\ Irene Esteban-Cornejo ${ }^{\mathrm{a}, \mathrm{d}}$, Jeremy A. Labrecque ${ }^{\mathrm{e}}$, Junwen Yang-Huang ${ }^{\mathrm{c}, \mathrm{f}}$, Hein Raat ${ }^{\mathrm{f}}$, \\ Meike W. Vernooij ${ }^{\mathrm{e}, \mathrm{g}}$, Tonya White ${ }^{\mathrm{b}, \mathrm{g}}$, Francisco B. Ortega ${ }^{\mathrm{a}}$, Henning Tiemeier ${ }^{\mathrm{b}, \mathrm{h}, *}$, \\ Ryan L. Muetzel ${ }^{\text {b,e }}$
}

a PROFITH "PROmoting FITness and Health through physical activity" research group, Sport and Health University Research Institute (iMUDS), Department of Physical and Sports Education, Faculty of Sport Sciences, University of Granada, Granada, Spain

${ }^{\mathrm{b}}$ Department of Child and Adolescent Psychiatry, Erasmus MC -Sophia Children's Hospital, Rotterdam, the Netherlands

' Generation R Study Group, Erasmus MC - Sophia Children's Hospital, Rotterdam, the Netherlands

${ }^{\mathrm{d}}$ Center for Cognitive and Brain Health, Department of Psychology, Northeastern University, Boston, MA, USA

e Department of Epidemiology, Erasmus MC, Rotterdam, the Netherlands

${ }^{\mathrm{f}}$ Department of Public Health, Erasmus MC, Rotterdam, the Netherlands

${ }^{g}$ Department of Radiology and Nuclear Medicine, Erasmus MC, Rotterdam, the Netherlands

${ }^{\mathrm{h}}$ Department of Social and Behavioral Science, Harvard T.H. Chan School of Public Health, Boston, MA, United States

\section{A R T I C L E I N F O}

\section{Keywords:}

MRI

Diffusion tensor imaging

Brain development

Sedentary behavior

Television viewing

Video games

Active commuting

\begin{abstract}
A B S T R A C T
Physical activity and sedentary behaviors have been linked to a variety of general health benefits and problems. However, few studies have examined how physical activity during childhood is related to brain development, with the majority of work to date focusing on cardio-metabolic health. This study examines the association between physical activity and screen time with white matter microstructure in the general pediatric population. In a sample of 2532 children ( $10.12 \pm 0.58$ years; 50.04\% boys) from the Generation R Study, a population-based cohort in Rotterdam, the Netherlands, we assessed physical activity and screen time using parent-reported questionnaires. Magnetic resonance imaging of white matter microstructure was conducted using diffusion tensor imaging. Total physical activity was positively associated with global fractional anisotropy $(\beta=0.057,95 \%$ $\mathrm{CI}=0.016,0.098, \mathrm{p}=0.007)$ and negatively associated with global mean diffusivity $(\beta=-0.079,95 \%$ $\mathrm{CI}=-0.120,-0.038, \mathrm{p}<0.001$ ), two commonly derived scalar measures of white matter microstructure. Two components of total physical activity, outdoor play and sport participation, were positively associated with global fractional anisotropy $(\beta=0.041,95 \% \mathrm{CI}=(0.000,0.083), \mathrm{p}=0.047 ; \beta=0.053,95 \% \mathrm{CI}=(0.010,0.096)$, $\mathrm{p}=0.015$, respectively) and inversely associated with global mean diffusivity $(\beta=-0.074,95 \% \mathrm{CI}=(-0.114$, $-0.033), \mathrm{p}<0.001 ; \beta=-0.043,95 \% \mathrm{CI}=(-0.086,0.000), \mathrm{p}=0.049$, respectively $)$. No associations were observed between screen time and white matter microstructure $(\mathrm{p}>0.05)$. This study provides new evidence that physical activity is modestly associated with white matter microstructure in children. In contrast, complementing other recent evidence on cognition, screen time was not associated with white matter microstructure. Causal inferences from these modest associations must be interpreted cautiously in the absence of longitudinal data. However, these data still offer a promising avenue for future work to explore to what extent physical activity may promote healthy white matter development.
\end{abstract}

Abbreviations: MRI, magnetic resonance imaging; METs, metabolic equivalents; DTI, diffusion tensor imaging; FA, fractional anisotropy; MD, mean diffusivity; RCT,

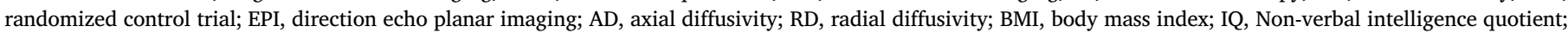

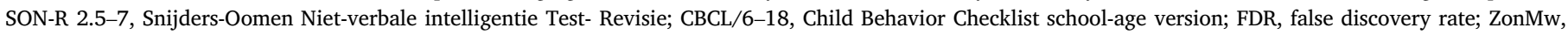
Netherlands Organization for Health Research and Development.

* Corresponding author. Department of Social and Behavioral Science, Harvard T.H. Chan School of Public Health Boston, Massachusetts, United States.

E-mail address: tiemeier@hsph.harvard.edu (H. Tiemeier). 


\section{Introduction}

Despite compelling evidence suggesting physical activity plays an important role in general health (Lee et al., 2012), a substantial proportion of youth do not meet the recommended levels of physical activity (Hallal et al., 2012). During childhood and adolescence, the brain undergoes a variety of developmental changes in white and gray matter structure (Lenroot and Giedd, 2006; Schmithorst and Yuan, 2010), which also coincide with changes in behavior and cognition. During this sensitive period of maturation, a number of environmental factors have been shown to be related to the brain's white matter macrostructure and microstructure (Frodl et al., 2012; Hanson et al., 2013; Bick and Nelson, 2016), yet little work has explored to what extent physical activity and sedentary behavior, associate with white matter in children.

Physical activity and sedentary behavior are the two independent behaviors that occupy all waking hours of a day. Physical activity, defined as any body movement that increases energy expenditure (e.g. active commuting, outdoor play, or sport participation) (Caspersen et al., 1985), has been consistently linked to mental health benefits across a person's lifespan (Biddle et al., 2018; Chekroud et al., 2018). On the other hand, sedentary behavior, defined as any waking behavior characterized by an energy expenditure $\leq 1.5$ metabolic equivalents (METs), while in a sitting, reclining or lying posture (Tremblay et al., 2017), has become a central component of the daily lives of children. Therefore, the debate about whether sedentary behavior, specifically recreational screen time, negatively impacts children mental health is becoming increasingly important. Recent work has suggested that while negative associations are observed between screen time (i.e., technology use and playing videogames) and mental health and cognition in young people, they are likely too small in magnitude to warrant policy change (Ferguson, 2017; Orben and Przybylski, 2019). Though previous work has studied the association of these two behaviors with indicators of mental health in childhood, little work has examined any neural correlates.

Magnetic resonance imaging (MRI) offers an in vivo view into the developing brain. White matter neuronal tissue consists of axons wrapped in the lipid-rich myelin sheath and is responsible for providing fast and efficient connections throughout cortex and subcortex. Diffusion tensor imaging (DTI) is able to sample features of the microstructural architecture of white matter. Fractional anisotropy (FA) and mean diffusivity (MD) are two commonly derived scalar metrics from DTI. FA represents the degree to which water diffuses preferentially along one axis (i.e., the diffusion is hindered by structures such as myelin and tightly packed axons), and has shown to increase with age during child and adolescent brain development (Giorgio et al., 2010; Schmithorst and Yuan, 2010; Tamnes et al., 2018) and in many cases to be lower in the context of various neurological and psychiatric diseases (Hulvershorn et al., 2011; Ayling et al., 2012; Aoki et al., 2018). MD is the simple average diffusion, with higher levels indicating relatively unimpeded diffusion (i.e., it is negatively correlated with FA). Numerous studies have demonstrated that developmental changes in white matter microstructure occur throughout childhood (Schmithorst and Yuan, 2010; Stiles and Jernigan, 2010), and the technique has been shown to be sensitive to subtle features of psychopathology (Muetzel et al., 2017). However, little work has explored whether physical activity or screen time are associated with white matter microstructure.

In one randomized control trial (RCT) of overweight 8-11 year-old children, the effect of an 8-month exercise intervention on white matter microstructure was examined (Krafft et al., 2014; Schaeffer et al., 2014). This study showed that FA increased in the uncinate and superior longitudinal fasciculus in the intervention group. Similarly, another RCT examined the effects of an after-school exercise program on the microstructure of white matter tracts in 7- to 9-year-old children. This study found that children who participated in the exercise program showed increased white matter microstructure in the genu of the corpus callosum
(Chaddock-Heyman et al., 2018). In addition, a cross-sectional study demonstrated that, in 9-to-10 year-old children, higher levels of cardiorespiratory fitness were associated with greater FA in sections of the corpus callosum, corona radiata, and superior longitudinal fasciculus (Chaddock-Heyman et al., 2014). However, greater cardiorespiratory fitness was also associated with lower FA in the corticospinal tract (Herting et al., 2014). Collectively, it is difficult to draw conclusions from this limited literature, which largely focuses on the relation between cardiorespiratory fitness, exercise and white matter microstructure, omitting the constructs of physical activity (e.g., outdoor play, active commuting, and sport participation). Further, to our knowledge, no other studies have examined whether screen time is associated with white matter microstructure in children.

The present study examined the associations of physical activity and screen time with white matter microstructure in 10-year old children from the general population. Based on previous literature, we hypothesized higher levels of physical activity would be related to higher FA and lower MD. We hypothesized increased screen time would be related to lower FA and higher MD, however, in line with previous literature (Ferguson, 2015, 2017; Orben and Przybylski, 2019), we expect these relationships to be relatively small.

\section{Materials and methods}

\subsection{Study design and participants}

The study was part of the Generation R Study, a prenatal populationbased cohort. A detailed description of the study design and methods has been published elsewhere (Kooijman et al., 2016). In total, 3992 children visited our study-dedicated imaging facility for an MRI scan at the mean age of 10 (White et al., 2018). Of the 3992 children, a total of 942 were excluded due to missing a complete DTI scan $(n=285)$, image artifacts $(n=37)$, poor image quality $(n=406)$, an incidental finding $(n=13)$ or their data were collected using different parameters on the MRI system $(n=201)$, leaving 3050 participants for analysis. After excluding children with missing information on physical activity $(n=518)$, the study sample included 2532 children (mean age $10.12 \pm 0.58$ years; $50.04 \%$ boys). Supplemental Table S1 shows the sample characteristics of children included in analyses of screen time $(\mathrm{N}=2346)$, which is highly similar to those included in analyses of physical activity. A flow chart illustrating the exclusion of data is depicted in Fig. 1. The Medical Ethics Committee of the Erasmus Medical Center approved the study procedures and participants provided written informed consent.

\subsection{Physical activity and screen time assessments}

Information on the level of physical activity and screen time were obtained through a parent-reported questionnaire administered when children were 10 years old. The questionnaires were intended for the primary caregiver who was most often the mother (97\%). To assess the level of physical activity, respondents indicated both the number of days per week and duration per day their child engages in: (i) walking or cycling to/from school, (ii) outdoor play, and (iii) sports. Time per week spent on each activity was calculated by using the following formula: weekly time spent on the activity $=$ (days per week) * (hours per day). A total physical activity score was calculated by adding the hours of active commuting, outdoor play, and sport participation per week.

Respondents were also asked to indicate the number of days and hours per day their child: (i) watches television (including videos/DVDs) and (ii) uses a computer or similar device (including video games). Screen times were assessed separately for weekdays and weekend days but were combined to estimate the total hours per week spent in each activity. A total weekly screen time score was calculated by adding the hours of playing video games and watching television. 


\section{3. $M R I$}

\subsubsection{Image acquisition}

MRI data were acquired with a 3 Tesla GE MR-750W system (General Electric, Milwaukee, WI). The DTI sequence consisted of a 35 direction echo planar imaging (EPI) sequence using the following sequence parameters: $\mathrm{TR}=12,500 \mathrm{~m} \mathrm{~s}, \quad \mathrm{TE}=72 \mathrm{~m} \mathrm{~s}, \quad$ flip angle $=90$, matrix $=120 \times 120, \quad F O V=240 \mathrm{~mm} \times 240 \mathrm{~mm}$, slice thickness $=2 \mathrm{~mm}$, number of slices $=65$ and voxel resolution $=2 \times 2 \times 2 \mathrm{~mm}^{3}$ (Muetzel et al., 2017).

\subsubsection{Image preprocessing}

Image preprocessing was conducted using the Functional MRI of the Brain's Software Library (Jenkinson et al., 2012) and the CAMINO toolkit (Cook et al., 2006) through python interfaces (https://fsl.fmrib.ox.ac.uk) (Gorgolewski et al., 2011). Images were first adjusted for minor head motion and eddy-current induced artifacts (Haselgrove and Moore, 1996; Jenkinson and Smith, 2001). In order to account for rotations applied to the image data (Leemans and Jones, 2009; Jones and Cercignani, 2010), the resulting transformation matrices were used to rotate the diffusion gradient direction table. Non-brain was removed using the FSL Brain Extraction Tool (Smith, 2002). The diffusion tensor was fit using the RESTORE method (Chang et al., 2005; Cook et al., 2006), and common scalar maps (i.e., FA, MD, Axial diffusivity (AD), radial diffusivity (RD)) were subsequently computed. Fully automated probabilistic tractography was run using a predefined series of seed- and target-masks from the FSL AutoPtx plugin (De Groot et al., 2015; Muetzel et al., 2017). Briefly, following a nonlinear registration of the FA map to standard space (FMRIB58 $1 \mathrm{~mm}$ ), a predefined library of seed, target, and exclusion masks were warped to each individual's native space FA map. FSL's Bayesian Estimation of Diffusion Parameters using Sampling Techniques (BEDPOSTx) and Probtrackx were used to conduct probabilistic fiber tractography, accounting for two fiber orientations (Behrens et al., 2003). Resulting connectivity distributions were normalized based on the total number of successful seed-to-target attempts, and were thresholded based on established values to remove voxels with a low probability (Muetzel et al., 2017). Average DTI scalar metrics were extracted for each tract (see Supplemental Material), with each voxel being weighted by the normalized connectivity value from probabilistic tractography. Global, whole brain estimates of DTI metrics (i.e., FA, MD, RD, AD) were calculated using a confirmatory factor analysis across multiple tracts described in detail elsewhere (Muetzel et al., 2017).

\subsubsection{Image quality assurance}

Raw image quality was assessed with both an automated software and with a visual inspection (Muetzel et al., 2017). Briefly, slice-wise variations in signal intensity were examined using the DTIprep package (htt ps://www.nitrc.org/projects/dtiprep). The sum-of-squares error (SSE)

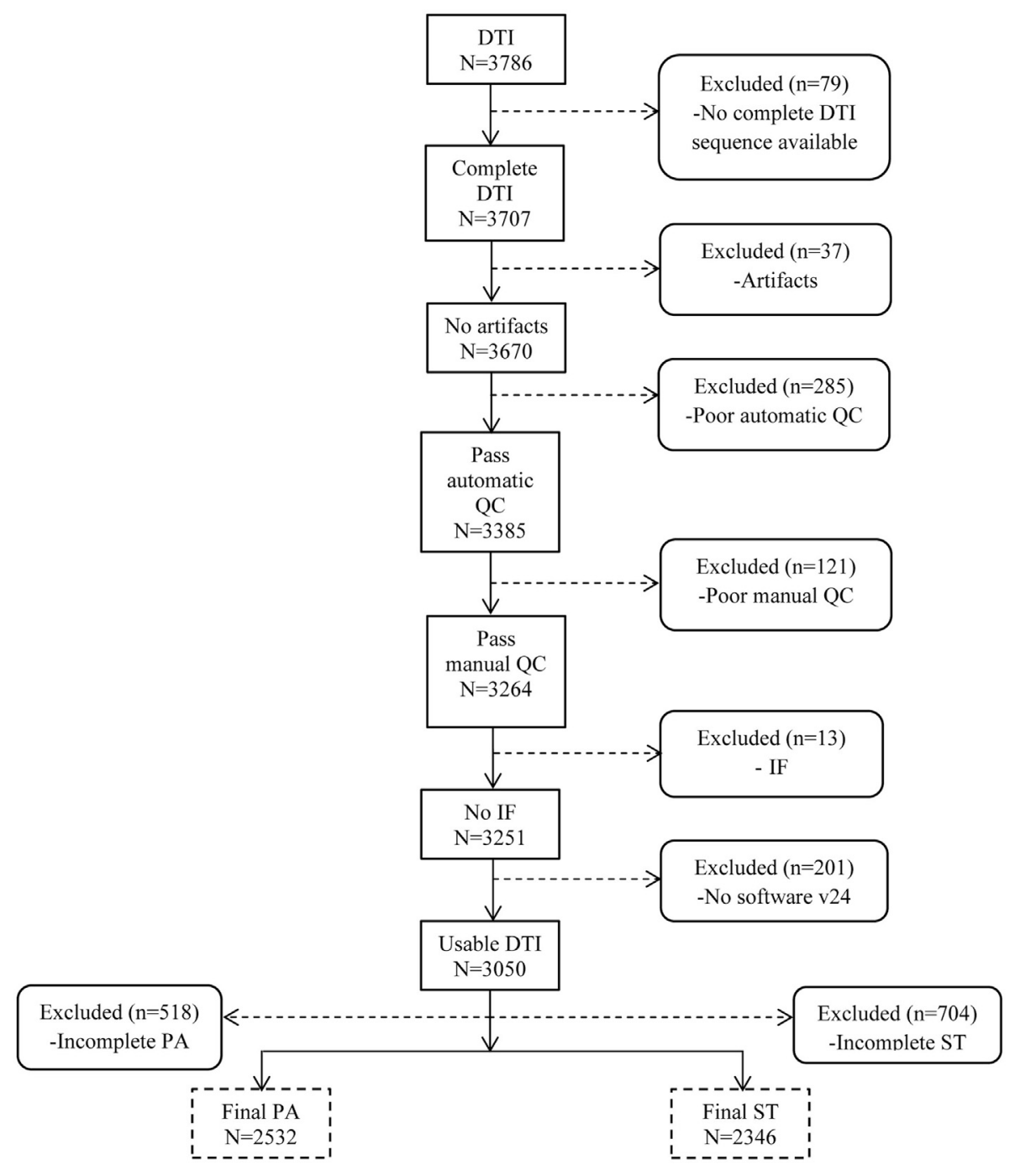

Fig. 1. Flow chart indication data inclusion/exclusion. Note: Box with dotted lines indicate the sample used in the current studys. QC $=$ quality control, IF $=$ incidental of finding, $\mathrm{PA}=$ physical activity, $\mathrm{ST}=$ screen time, $\mathrm{MRI}=$ magnetic resonance imaging, $\mathrm{DTI}=$ diffusion tensor imaging. 
maps from the tensor estimation were also calculated and visually inspected for structured noise. Lastly, nonlinear registration to standard space was inspected for accuracy to ensure seed and target masks were properly aligned to native space. Datasets determined to be of insufficient quality for statistical analyses were excluded $(n=285$ from automated quality control of slice-wise variation in signal and $n=121$ from an additional visual inspection of SSE maps and registration quality). Example images of FA maps from children representative of the sample are presented in the Supplementary Material (see Fig. S1).

\subsection{Covariates}

Age at MRI scanning and sex were included as covariates. Further, maternal education level and ethnicity were assessed by questionnaires. Ethnicity was based on the country of birth of the mother and mother's parents. Maternal education level was defined by the highest completed education and divided into 3 categories ranging from low (from no education to primary school), middle (high school or vocational training) to high education level (from higher vocational education to university). Height and weight were measured at the age of 10 years at the research center and body mass index (BMI) was calculated using standardized scores according to the Dutch reference growth curves (https://growtha nalyser.org) (Fredriks et al., 2000). A non-verbal intelligence quotient (IQ) was assessed at approximately 6 years of age using the Snijders-Oomen Niet-verbale intelligentie Test- Revisie (SON-R 2.5-7) (Tellegen et al., 2005). Emotional and behavioral problems were assessed at age of 10 years by parent-report using the validated Child Behavior Checklist school-age version (CBCL/6-18) (Achenbach and Rescorla, 2003). Apart from non-verbal IQ (12\%), the percentage of missing values did not exceed $10 \%$.

\subsection{Statistical analysis}

All analyses were performed using the Statistical Package for Social Sciences (IBM SPSS Statistics for Windows, version 22.0, Armonk, NY, p set at $<0.05$ ). Multiple imputation was used to impute missing data of covariates. In total, 10 imputed datasets were created and pooled results are presented. Multiple linear regression analyses were performed with physical activity or screen time measures as independent variables and DTI scalar metrics (i.e., FA, and MD) as dependent variables. Independently, as they provide added information in the interpretation of the underlying white matter microstructure, we also tested the association of physical activity and screen time variables with global RD and global AD. Linear regression analyses were hierarchically adjusted for covariates by creating two models. Model 1 included age at the time of scanning, sex, ethnicity, maternal education, and BMI. Model 2 was additionally adjusted for behavior problems and non-verbal IQ. In order to determine whether the association with white matter microstructure was indeed only global or restricted to a particular set of white matter bundles, associations with FA and MD within individual tracts were also tested if exposures showed an association with global DTI metrics. For tractspecific analyses, false discovery rate (FDR) correction was applied to account for the number of tests performed.

Additionally, a number of sensitivity analyses were run. First, total physical activity and total screen time were entered into the model simultaneously to adjust estimates for one another. Second, we divided the total sample in four subgroups of participants according to their physical activity and screen time levels to compare global FA and MD amongst the groups. Physical activity and screen time groups were defined according to the sample median (high/low physical activity/ screen time). Third, different interaction terms were tested to ascertain whether associations were different across sub-groups of subjects (i.e., sex, parental educational level, ancestral background). Lastly, we tested quadratic and cubic age terms, as well as an age-by-sex interaction term (using a quadratic age term). We also tested quadratic screen time and physical activity term, as well as the interactions between quadratic total physical activity variable and quadratic total screen time variable in association with white matter microstructure.

\section{Results}

Table 1 presents participant demographic characteristics. Differences between physical activity and screen time levels between boys and girls are shown in Table S2. Characteristics of included and excluded participants are shown in Table S3.

\subsection{Association of physical activity and screen time with global white matter microstructure}

Total physical activity was positively associated with global FA ( $\beta=0.051,95 \% \mathrm{CI}=(0.010,0.092), \mathrm{p}=0.016)$ when the model was adjusted for sociodemographic factors. Further, the positive association between physical and global FA remained after additional adjustment for behavior problems and non-verbal IQ $(\beta=0.057,95 \% \mathrm{CI}=(0.016$, 0.098), $\mathrm{p}=0.007$ ). Results from analyses of global FA and MD are presented in Table 2, and follow-up analyses with global RD and AD to better describe the diffusion profile are presented in Table S4. To determine whether a particular type of physical activity (i.e. active commuting, outdoor play and sport participation) was responsible for the total PA association with global FA, each type of physical activity was examined separately. In the fully adjusted model (model 2), positive associations between outdoor play and global FA $(\beta=0.041,95 \% \mathrm{CI}=(0.000,0.083)$, $\mathrm{p}=0.047)$ and between sport participation and global FA were observed $(\beta=0.053,95 \% \mathrm{CI}=(0.010,0.096), \mathrm{p}=0.015)$.

Total physical activity was inversely associated with global MD ( $\beta=-0.079,95 \% \mathrm{CI}=(-0.119,-0.038), \mathrm{p}<0.001)$ when the model was adjusted for sociodemographic covariates (model 1). After, additional adjustment for behavioral problems and non-verbal IQ the association remained similar (model 2) $(\beta=-0.079,95 \% \mathrm{CI}=(-0.120$, $-0.038), p<0.001$ ). Each type of physical activity was examined separately to determine whether all or a subset of activities were responsible for the associations with total physical activity. In the fully adjusted

Table 1

Sample characteristics.

\begin{tabular}{|c|c|c|c|c|}
\hline & $\mathrm{N}$ & Mean/\% & & SD \\
\hline \multicolumn{5}{|l|}{ Sex } \\
\hline Boys, $\%$ & 1267 & 50.04 & & \\
\hline Age at MRI assessment,years & 2532 & 10.12 & & 0.58 \\
\hline Age at CBCL assessment, years & 2495 & 9.71 & & 0.28 \\
\hline \multicolumn{5}{|l|}{ Ethnicity, \% } \\
\hline Dutch & 1671 & 66.63 & & \\
\hline Other Western & 213 & 8.49 & & \\
\hline Non-Western & 624 & 24.88 & & \\
\hline BMI, $\mathrm{kg} / \mathrm{m}^{2}$ & 2526 & 17.36 & & 2.50 \\
\hline Behavior problems, sum score CBCL & 2475 & 16.76 & & 14.63 \\
\hline Non-verbal IQ, sum score SON-R & 2218 & 104.32 & & 14.59 \\
\hline \multicolumn{5}{|l|}{ Maternal education, $\%$} \\
\hline Low & 187 & 8.06 & & \\
\hline Medium & 618 & 26.65 & & \\
\hline High & 1514 & 65.29 & & \\
\hline \multicolumn{5}{|l|}{ Exposures characteristics } \\
\hline Total physical activity, h/w & 2532 & 9.06 & 5.10 & \\
\hline Total screen time, $\mathrm{h} / \mathrm{w}$ & 2346 & 17.10 & 11.63 & \\
\hline
\end{tabular}

*Note: 86 participants had no data on physical activity but they were included in analyses of screen time. MRI = magnetic resonance imaging, $\mathrm{CBCL}=$ Child Behavior Checklist school-age, BMI= Body mass index, $\mathrm{IQ}=$ intelligence quotient, $\mathrm{SON}-\mathrm{R}=$ the Snijders-Oomen Niet-verbale intelligentie Test- Revisie, $\mathrm{h} / \mathrm{w}$ $=$ hours per week. Total physical activity = hours of active commuting, outdoor play, and sport participation per week. Total screen time $=$ hours of computer time, playing video games, and watching television. Maternal education level was defined by the highest completed education and divided into three categories ranging from low (from no education to primary school), middle (high school or vocational training) to high education level (from higher vocational education to university). 
Association between physical activity and screen time with global fractional anisotropy and mean diffusivity.

\begin{tabular}{|c|c|c|c|c|c|c|}
\hline & \multicolumn{6}{|c|}{ Fractional anisotropy } \\
\hline & \multicolumn{3}{|l|}{ Model 1} & \multicolumn{3}{|l|}{ Model 2} \\
\hline & $\beta$ & $95 \% \mathrm{CI}$ & $\mathrm{p}$ & $\beta$ & CI & $\mathrm{p}$ \\
\hline \multicolumn{7}{|l|}{ Physical activity $(\mathrm{n}=2532)$} \\
\hline Total physical activity, h/w & 0.051 & $(0.010,0.092)$ & 0.016 & 0.057 & $(0.016,0.098)$ & 0.007 \\
\hline Active commuting, $\mathrm{h} / \mathrm{w}$ & 0.021 & $(-0.020,0.062)$ & 0.325 & 0.023 & $(-0.018,0.064)$ & 0.272 \\
\hline Outdoor play, h/w & 0.035 & $(-0.006,0.076)$ & 0.093 & 0.041 & $(0.000,0.083)$ & 0.047 \\
\hline Sport participation, $\mathrm{h} / \mathrm{w}$ & 0.052 & $(0.009,0.094)$ & 0.017 & 0.053 & $(0.010,0.096)$ & 0.015 \\
\hline \multicolumn{7}{|l|}{ Screen time $(n=2346)$} \\
\hline Total screen time, $\mathrm{h} / \mathrm{w}$ & -0.021 & $(-0.065,0.023)$ & 0.347 & -0.010 & $(-0.054,0.035)$ & 0.668 \\
\hline Playing computer games, $\mathrm{h} / \mathrm{w}$ & -0.003 & $(-0.045,0.039)$ & 0.880 & 0.000 & $(-0.041,0.042)$ & 0.985 \\
\hline \multirow[t]{4}{*}{ Watching television, $\mathrm{h} / \mathrm{w}$} & -0.028 & $(-0.073,0.017)$ & 0.219 & -0.014 & $(-0.060,0.031)$ & 0.532 \\
\hline & \multicolumn{6}{|c|}{ Mean diffusivity } \\
\hline & \multicolumn{3}{|l|}{ Model 1} & \multicolumn{3}{|l|}{ Model 2} \\
\hline & $\beta$ & $95 \%$ CI & $\mathrm{p}$ & $\beta$ & CI & $\mathrm{p}$ \\
\hline \multicolumn{7}{|l|}{ Physical activity $(\mathrm{n}=2532)$} \\
\hline Total physical activity, h/w & -0.079 & $(-0.119,-0.038)$ & $<0.001$ & -0.079 & $(-0.120,-0.038)$ & $<0.001$ \\
\hline Active commuting, $\mathrm{h} / \mathrm{w}$ & -0.005 & $(-0.045,0.036)$ & 0.821 & -0.005 & $(-0.046,0.036)$ & 0.812 \\
\hline Outdoor play, h/w & -0.073 & $(-0.114,-0.032)$ & $<0.001$ & -0.074 & $(-0.114,-0.033)$ & $<0.001$ \\
\hline Sport participation, $\mathrm{h} / \mathrm{w}$ & -0.043 & $(-0.086,-0.001)$ & 0.045 & -0.043 & $(-0.086,0.000)$ & 0.049 \\
\hline \multicolumn{7}{|l|}{ Screen time $(\mathrm{n}=2346)$} \\
\hline Total screen time, $\mathrm{h} / \mathrm{w}$ & -0.028 & $(-0.072,0.015)$ & 0.203 & -0.031 & $(-0.075,0.013)$ & 0.170 \\
\hline Playing computer games, $\mathrm{h} / \mathrm{w}$ & -0.024 & $(-0.066,0.017)$ & 0.250 & -0.026 & $(-0.067,0.016)$ & 0.230 \\
\hline Watching television, $\mathrm{h} / \mathrm{w}$ & -0.022 & $(-0.067,0.022)$ & 0.325 & -0.025 & $(-0.070,0.020)$ & 0.280 \\
\hline
\end{tabular}

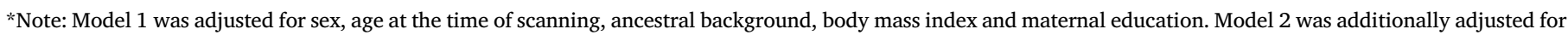
emotional and behavior problems and non-verbal intelligence quotient. $\mathrm{h} / \mathrm{w}=$ hours per week.

model (model 2), outdoor play and sport participation were inversely associated with $\mathrm{MD}(\beta=-0.074,95 \% \mathrm{CI}=(-0.114,-0.033), \mathrm{p}<0.001$; $\beta=-0.043,95 \% \mathrm{CI}=(-0.086,0.000), \mathrm{p}=0.049$, respectively $)$. In order to better describe these associations with $\mathrm{FA}$ and $\mathrm{MD}$, further analyses explored which underlying diffusivity metrics (i.e., $\mathrm{RD}$ and/or $\mathrm{AD}$ ) were associated with physical activity. Both $\mathrm{RD}$ and $\mathrm{AD}$ were significantly associated with total physical activity $(\beta=-0.079,95 \% \mathrm{CI}=(-0.120$, $-0.039), \mathrm{p}<0.001 ; \beta=-0.054,95 \% \mathrm{CI}=(-0.094,0.014), \mathrm{p}=0.008$, respectively).

No association was found between individual or aggregate screen time variables and global DTI metrics (all p >0.05) (Table 2).

\subsection{Association between physical activity, screen time and tract-specific FA and $M D$}

Associations between total physical activity and tract-specific FA and MD are shown in Table S5. Briefly, no association was found between total physical activity and FA within individual tracts when significance levels were adjusted for multiple testing. However, total physical activity was associated with MD in nearly all tracts when adjusted for multiple comparisons. Fig. 2 graphically represents the significant negative associations between total physical activity and MD across individual tracts.

Lastly, exploratory analyses testing the association between total screen time and tract-specific FA and MD are shown in Table S6.

\subsection{Sensitivity analyses}

Results remained similar when models for physical activity and global white matter microstructure were additionally adjusted for total screen time, suggesting that physical activity is positively associated with white matter microstructure, independent of total screen time (Table S7). Screen time was still unrelated to global DTI metrics after additional adjustment for total physical activity (Table S7).

Next, when categorically subdividing the sample based on physical activity and screen time levels (e.g., high screen time, low physical activity), the subgroup with high physical activity ( $>8.4 \mathrm{~h} /$ week) and high screen time ( $>14.8 \mathrm{~h} /$ week seem) showed lower MD compared to the rest of subgroups (Fig. S2 and Fig. S3, respectively).

Then, different interaction terms were tested to ascertain whether associations were different across sub-groups of subjects. The association between physical activity and white matter microstructure was consistent across parents' education categories. Similarly, no interaction between sex or ethnicity and physical activity was observed.

Lastly, we have included into the model nonlinear age terms, specifically a quadratic age term (Table S8) and also included a cubic age term and results remain highly similar (Table S9). We also tested age-bysex interaction effects with these variables (i.e., age $^{2}$ and age $^{3}$ ) and the interaction term was not significant (all $\mathrm{P}>0.470$ ). Importantly, the model estimates for the association with physical activity and screen time also remained highly similar. Finally, nonlinear screen time and physical activity terms were not associated with FA or MD (See Supplemental Material).

\section{Discussion}

Using neuroimaging data from over 2500 children, we found that physical activity is associated with white matter microstructure, specifically outdoor play and sport participation time. On the other hand, no association was observed between screen time and white matter microstructure. In the context of the literature, this study expands upon the compelling evidence of general health benefits of physical activity by demonstrating a new association with white matter microstructure in children.

Previous work on typical brain development has shown a positive association between FA and age, as well as a negative association with MD (Barnea-Goraly et al., 2005; Eluvathingal et al., 2007; Asato et al., 2010; Giorgio et al., 2010; Schmithorst and Yuan, 2010; Brouwer et al., 2012; Tamnes et al., 2018). Further, a similar pattern of association has been shown with cognitive function, where white matter microstructure is associated with better neuropsychological performance (Muetzel et al., 2015). Within this context, the positive link between physical activity and white matter microstructure shown in the present study could explain the improvements observed in cognition (Hillman et al., 2008, 2015). Importantly, specific aspects of physical activity, namely outdoor 


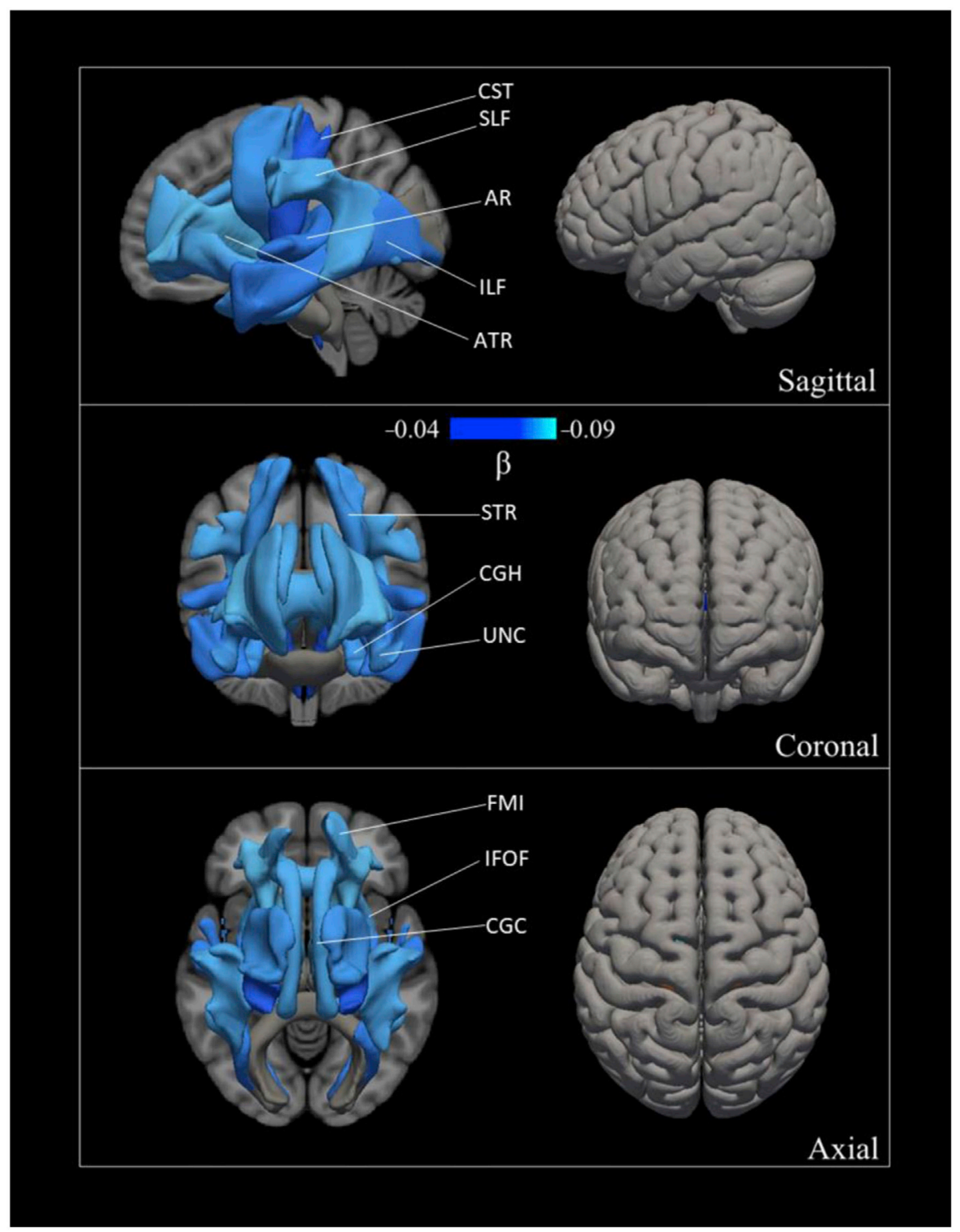

Fig. 2. Association between total physical activity and tract-specific MD. Colour bar represents standardized beta coefficients with lighter blue referring to negative association. Lighter blue referring to stronger association between total physical activity and MD. Corticospinal tract (CST), acoustic radiation (AR), anterior thalamic radiation (ATR), superior longitudinal fasciculus (SLF), inferior longitudinal fasciculus (ILF), inferior fronto-occipital fasciculus (IFOF), uncinate fasciculus (UNC), cingulate gyrus part of cingulum (CGC), hippocampal part of the cingulum (CGH), forceps minor (FMI), and superior thalamic radiations (STR).

play and sport participation, were associated with white matter microstructure. The association between physical activity and white matter microstructure has not been studied previously, which hampers further comparisons with similar studies. Nonetheless, this work compliments previous studies focused on the association between cardiorespiratory fitness and exercise with white matter microstructure. For instance, previous studies demonstrated that, in 9-to-10 year-old children, higher levels of cardiorespiratory fitness were associated with higher FA in sections of the corpus callosum, corona radiata, and superior longitudinal fasciculus (Chaddock-Heyman et al., 2014). In contrast, adolescents with better cardiorespiratory fitness showed greater number of streamlines, especially in the corticospinal tract and forceps minor (Herting et al., 2014), but also lower FA in the corticospinal tract (Herting et al., 2014). Additionally, an 8-month exercise intervention showed increased FA in sections of the uncinate fasciculus and superior longitudinal fasciculus in overweight children in the exercise group when compared to controls (Krafft et al., 2014; Schaeffer et al., 2014). Moreover, an 9-month exercise intervention showed increased white matter microstructure in the genu of the corpus callosum in 7- to 9-year-old children in the exercise group when compared to controls (Chaddock-Heyman et al., 2018).

In the present study, no association was found between active commuting and white matter microstructure. Previous work has also shown that active commuting was not associated with cognitive performance in children (Van Dijk et al., 2014). Thus, results are in line with an increasing literature suggesting that increases in cognitive functioning in childhood due to physical activity are most clearly observed in tasks that involve executive functioning (e.g., sport participation) (Tomporowski et al., 2008).

Previous work has demonstrated that increased levels of physical activity might improve cerebral blood flow (Hillman et al., 2008), and DTI has proven to be sensitive to white matter microstructural changes that may result from cardio- and neuro-vascular risk factors (Lee et al., 2009; Shefer et al., 2013). A possible physiological mechanism could be the positive influence physical activity has on cardio-metabolic risk factors, such as insulin resistance, blood lipids, blood pressure and inflammatory proteins (Eisenmann, 2004). Moreover, previous literature showed higher levels physical activity are associated with better mental health in children (Biddle and Asare, 2011; Biddle et al., 2018). As childhood is a period where many physiological and psychological changes occur, public health advocation for novel approaches to increasing physical activity in children could be useful for improved (neuro) physiological and psychological health. In this line, a recent large 
study of physical activity and cognition showed higher levels of physical activity were associated with better global cognition (Walsh et al., 2018). Our results suggest that these improvements in cognition could be facilitated by better underlying white matter microstructure.

Another important finding was that screen time was not associated with white matter microstructure. To the best of our knowledge, this is the first study to examine the association between screen time and white matter microstructure in children. These results further support the work from Ferguson et al. and Orben et al. (Ferguson, 2017; Orben and Przybylski, 2019) who showed that the relationship between screen time and different mental health outcomes (e.g., externalizing problems, internalizing problems and academic performance) was null or very small. Overall, the association between screen time and mental health are likely nonlinear, and moderate engagement in certain screen time activities may not lead to behavioral or emotional problems (Ferguson, 2017). For instance, previous research found that meeting the $2 \mathrm{~h}$ or less recreational screen time per day recommendations was associated with superior global cognition (Walsh et al., 2018). In contrast, frequent weekly use of video games was associated with conduct problems, as well as, increased levels of television viewing were negatively associated with neurocognitive development of children (Takeuchi et al., 2015). In addition to these discrepancies in the literature, advances in technology (e.g., availability of hand-held media devices, and social media) compared to the construct of purely passive television viewing, further underscore the importance of maintaining well-characterized measures of screen time, as differing amounts/types of screen time could influence the brain differently. Interestingly, associations between physical activity and white matter remained after adjusting for levels of screen time. Thus, though purely speculative, the benefits of physical activity may not be negatively influenced by playing computer games and watching television moderately, which has been reported previously for other general cardiometabolic outcomes (Rezende et al., 2014).

Key strengths of the current study are the large sample size, and incorporation of population-based neuroimaging. The nature and size of the cohort allocates us with the data and power to adjust for multiple confounding factors. Further, the population-based sampling offers increased generalizability of the findings compared to previous studies. However, several limitations require discussion. First, this is a crosssectional study, limiting inferences about causality and directionality to any of the associated factors. Further, though residual confounding remains a possibility, effect estimates were not substantially changed after adjusting for a number of potential confounding factors. Future work should consider other environmental confounders including wellcharacterized measures of socioeconomic status. Second, physical activity and screen time levels were assessed by parental-reported questionnaires, lending to the possibility of under- or overestimations of the behaviors. Therefore, objective measures such as accelerometry and experience sampling should be utilized in future studies. Lastly, the effect sizes for the association between physical activity and white matter microstructure are relatively small. However, similar effect sizes are observed in structure-function associations with cognition and white matter microstructure (Muetzel et al., 2015), and the clinical relevance of such effect sizes in the context of DTI has yet to be formally evaluated.

\section{Conclusion}

This study demonstrates that higher levels of physical activity are associated with greater white matter microstructure in children ages 9to-10 years old from the general population. No association was observed between screen time and white matter microstructure. Future work should continue to explore longitudinal data in order to more concretely decipher the temporality of the associations, so that educational and health institutions can consider whether to promote physical activity during childhood as a potential (modifiable) protective factor in the context of brain health.

\section{Funding}

This study was supported by the Sophia Foundation (S18-20 awarded to RM) and Netherlands Organization for Health Research and Development (ZonMw) (project 016.VICI.170.200, awarded to HT and TOP 91211021, awarded to TW). Supercomputing resources were supported by the Netherlands Organization for Scientific Research (Exacte Wetenschappen) and SURFsara (Cartesius Compute Cluster, www.surfsara.nl). The Generation R Study is conducted by the Erasmus Medical Center in close collaboration with the School of Law and the Faculty of Social Sciences of the Erasmus University Rotterdam, the Municipal Health Service Rotterdam area, and the Stichting Trombosedienst en Artsenlaboratorium Rijnmond. The general design of the Generation R Study was made possible by financial support from the Erasmus Medical Center, Rotterdam, ZonMw, the Netherlands Organization for Scientific Research, and the Ministry of Health, Welfare, and Sport. The authors gratefully acknowledge the contributions of the participating children and parents, general practitioners, hospitals, midwives, and pharmacies in Rotterdam. MRA received a scholarship from the University of Granada from the PhD International Mobility Program for a brief stay in the Erasmus Medical Centre in Rotterdam (The Netherlands). IEC is supported by a grant from the Alicia Koplowitz Foundation. This work is part of the María Rodriguez-Ayllon Ph.D. Thesis conducted in the Biomedicine Doctoral Studies of the University of Granada, Spain.

\section{Acknowledgments}

The authors thank children and parents who participated in this study. The authors report no biomedical financial interests or potential conflicts of interest.

\section{Appendix A. Supplementary data}

Supplementary data to this article can be found online at https://doi. org/10.1016/j.neuroimage.2019.116258.

\section{References}

Achenback, T.M., Rescorla, L a, 2003. Reliability, internal consistency, cross-informant agreement, and stability. Man. ASEBA Sch. Age Forms Profiles 99- 107.

Aoki, Y., Cortese, S., Castellanos, F.X., 2018. Research Review: diffusion tensor imaging studies of attention-deficit/hyperactivity disorder: meta-analyses and reflections on head motion. J. Child Psychol. Psychiatry 59, 193-202.

Asato, M.R., Terwilliger, R., Woo, J., Luna, B., 2010. White matter development in adolescence: a DTI study. Cerebr. Cortex 20, 2122-2131.

Ayling, E., Aghajani, M., Fouche, J.P., Van Der Wee, N., 2012. Diffusion tensor imaging in anxiety disorders. Curr. Psychiatr. Rep. 14, 197-202.

Barnea-Goraly, N., Menon, V., Eckert, M., Tamm, L., Bammer, R., Karchemskiy, A., et al., 2005. White matter development during childhood and adolescence: a cross-sectional diffusion tensor imaging study. Cerebr. Cortex 15, 1848-1854.

Behrens, T.E.J., Woolrich, M.W., Jenkinson, M., Johansen-Berg, H., Nunes, R.G., Clare, S., et al., 2003. Characterization and propagation of uncertainty in diffusion-weighted MR imaging. Magn. Reson. Med. 50, 1077-1088.

Bick, J., Nelson, C.A., 2016. Early adverse experiences and the developing brain. Neuropsychopharmacology 41, 177-196.

Biddle, S.J.H., Asare, M., 2011. Physical activity and mental health in children and adolescents: a review of reviews. Br. J. Sports Med. 45, 886-895.

Biddle, S.J.H., Ciaccioni, S., Thomas, G., Vergeer, I., 2018. Physical activity and mental health in children and adolescents: an updated review of reviews and an analysis of causality. Psychol. Sport Exerc. 1-10.

Brouwer, R.M., Mandl, R.C.W., Schnack, H.G., Soelen, ILC va, Baal, GC van, Peper, J.S., et al., 2012. White matter development in early puberty: a longitudinal volumetric and diffusion tensor imaging twin study. PLoS One 7, e32316.

Caspersen, C.J., Powell, K.E., Christenson, G.M., 1985. Physical activity, exercise, and physical fitness: definitions and distinctions for health-related research. Public Health Rep. 100, 126-131.

Chaddock-Heyman, L., Erickson, K.I., Holtrop, J.L., Voss, M.W., Pontifex, M.B., Raine, L.B., et al., 2014. Aerobic fitness is associated with greater white matter integrity in children. Front. Hum. Neurosci. 8, 584.

Chaddock-Heyman, L., Erickson, K.I., Kienzler, C., Drollette, E., Raine, L., Kao, S.-C., et al., 2018. Physical activity increases white matter microstructure in children. Front. Neurosci. 12, 950 . 
Chang, L.C., Jones, D.K., Pierpaoli, C., 2005. RESTORE: robust estimation of tensors by outlier rejection. Magn. Reson. Med. 53, 1088-1095.

Chekroud, S.R., Gueorguieva, R., Zheutlin, A.B., Paulus, M., Krumholz, H.M., Krystal, J.H., et al., 2018. Association between physical exercise and mental health in 1.2 million individuals in the USA between 2011 and 2015: a cross-sectional study. The Lancet Psychiatr. 5, 739-746.

Cook, P a, Bai, Y., Seunarine, K.K., Hall, M.G., Parker, G.J., Alexander, D.C., 2006. Camino: open- source diffusion-MRI reconstruction and processing. 14th Sci. Meet. Int. Soc. Magn. Reson. Med. 14, 2759.

Van Dijk, M.L., De Groot, R.H., Van Acker, F., Savelberg, H.H., Kirschner, P.A., 2014. Active commuting to school, cognitive performance, and academic achievement: an observational study in Dutch adolescents using accelerometers. BMC Public Health 14, 799.

Eisenmann, J.C., 2004. Physical activity and cardiovascular disease risk factors in children and adolescents: an overview. Can. J. Cardiol. 20, 295-301.

Eluvathingal, T.J., Hasan, K.M., Kramer, L., Fletcher, J.M., Ewing-Cobbs, L., 2007. Quantitative diffusion tensor tractography of association and projection fibers in normally developing children and adolescents. Cerebr. Cortex 17, 2760-2768.

Ferguson, C.J., 2015. Do angry birds make for angry children? A meta-analysis of video game influences on children's and adolescents' aggression, mental health, prosocial behavior, and academic performance. Perspect. Psychol. Sci. 10, 646-666.

Ferguson, C.J., 2017. Everything in moderation: moderate use of screens unassociated with child behavior problems. Psychiatr. Q. 88, 797-805.

Fredriks, A.M., van Buuren, S., Burgmeijer, R.J., Meulmeester, J.F., Beuker, R.J., Brugman, E., et al., 2000. Continuing positive secular growth change in The Netherlands 1955-1997. Pediatr. Res. 47, 316-323.

Frodl, T., Carballedo, A., Fagan, A.J., Lisiecka, D., Ferguson, Y., Meaney, J.F., 2012. Effects of early- life adversity on white matter diffusivity changes in patients at risk for major depression. J. Psychiatry Neurosci. 37, 37-45.

Giorgio, A., Watkins, K.E., Chadwick, M., James, S., Winmill, L., Douaud, G., et al., 2010 Longitudinal changes in grey and white matter during adolescence. Neuroimage 49, 94-103.

Gorgolewski, K., Burns, C.D., Madison, C., Clark, D., Halchenko, Y.O., Waskom, M.L., et al., 2011. Nipype: a flexible, lightweight and extensible neuroimaging data processing framework in Python. Front. Neuroinf. 5, 13.

De Groot, M., Ikram, M.A., Akoudad, S., Krestin, G.P., Hofman, A., Van Der Lugt, A., et al., 2015. Tract-specific white matter degeneration in aging: the Rotterdam study. Alzheimer's Dementia 11, 321-330.

Hallal, P.C., Andersen, L.B., Bull, F.C., Guthold, R., Haskell, W., Ekelund, U., et al., 2012 Global physical activity levels: surveillance progress, pitfalls, and prospects. Lancet 380, 247-257.

Hanson, J.L., Adluru, N., Chung, M.K., Alexander, A.L., Davidson, R.J., Pollak, S.D., 2013. Early neglect is associated with alterations in white matter integrity and cognitive functioning. Child Dev. 84, 1566-1578.

Haselgrove, J.C., Moore, J.R., 1996. Correction for distortion of echo-planar images used to calculate the apparent diffusion coefficient. Magn. Reson. Med. 36, 960-964.

Herting, M.M., Colby, J.B., Sowell, E.R., Nagel, B.J., 2014. White matter connectivity and aerobic fitness in male adolescents. Dev. Cogn. Neurosci. 7, 65-75.

Hillman, C.H., Erickson, K.I., Kramer, A.F., 2008. Be smart, exercise your heart: exercise effects on brain and cognition. Nat. Rev. Neurosci. 9, 58-65.

Hillman, C.H., Khan, N.A., Kao, S.-C., 2015. The relationship of health behaviors to childhood cognition and brain health. Ann. Nutr. Metab. 66 (Suppl. 3), 1-4.

Hulvershorn, L.A., Cullen, K., Anand, A., 2011. Toward dysfunctional connectivity: a review of neuroimaging findings in pediatric major depressive disorder. Brain Imaging Behav. 5, 307-328.

Jenkinson, M., Beckmann, C.F., Behrens, T.E.J., Woolrich, M.W., Smith, S.M., 2012. FSL. Neuroimage 62, 782-790.

Jenkinson, M., Smith, S., 2001. A global optimisation method for robust affine registration of brain images. Med. Image Anal. 5, 143-156.

Jones, D.K., Cercignani, M., 2010. Twenty-five pitfalls in the analysis of diffusion MRI data. NMR Biomed. 23, 803-820.
Kooijman, M.N., Kruithof, C.J., van Duijn, C.M., Duijts, L., Franco, O.H., van IJzendoorn, M.H., et al., 2016. The Generation R Study: design and cohort update 2017. Eur. J. Epidemiol. 31, 1243-1264.

Krafft, C.E., Schaeffer, D.J., Schwarz, N.F., Chi, L., Weinberger, A.L., Pierce, J.E., et al. 2014. Improved frontoparietal white matter integrity in overweight children is associated with attendance at an after-school exercise program. Dev. Neurosci. 36, $1-9$.

Lee, D.Y., Fletcher, E., Martinez, O., Ortega, M., Zozulya, N., Kim, J., et al., 2009. Regional pattern of white matter microstructural changes in normal aging, MCI, and AD. Neurology 73, 1722-1728.

Lee, I.-M., Shiroma, E.J., Lobelo, F., Puska, P., Blair, S.N., Katzmarzyk, P.T., et al., 2012. Effect of physical inactivity on major non-communicable diseases worldwide: an analysis of burden of disease and life expectancy. Lancet 380, 219-229.

Leemans, A., Jones, D.K., 2009. The B-matrix must be rotated when correcting for subject motion in DTI data. Magn. Reson. Med. 61, 1336-1349.

Lenroot, R.K., Giedd, J.N., 2006. Brain development in children and adolescents: insights from anatomical magnetic resonance imaging. Neurosci. Biobehav. Rev. 30, 718-729.

Muetzel, R.L., Blanken, L.M.E., van der Ende, J., El Marroun, H., Shaw, P., Sudre, G., et al., 2017. Tracking brain development and dimensional psychiatric symptoms in children: a longitudinal population-based neuroimaging study. Am. J. Psychiatry $175,54-62$.

Muetzel, R.L., Mous, S.E., van der Ende, J., Blanken, L.M.E., van der Lugt, A., Jaddoe, V.W.V., et al., 2015. White matter integrity and cognitive performance in school-age children: a population-based neuroimaging study. Neuroimage 119, $119-128$.

Orben, A., Przybylski, A.K., 2019. The association between adolescent well-being and digital technology use. Nat. Hum. Behav. 3, 173-182.

Rezende, LFM de, Rodrigues Lopes, M., Rey-López, J.P., Matsudo, V.K.R., Luiz, O. do C., 2014. Sedentary behavior and health outcomes: an overview of systematic reviews. PLoS One 9, e105620.

Schaeffer, D.J., Krafft, C.E., Schwarz, N.F., Chi, L., Rodrigue, A.L., Pierce, J.E., et al., 2014. An 8- month exercise intervention alters frontotemporal white matter integrity in overweight children. Psychophysiology 51, 728-733.

Schmithorst, V.J., Yuan, W., 2010. White matter development during adolescence as shown by diffusion MRI. Brain Cogn. 72, 16-25.

Shefer, G., Marcus, Y., Stern, N., 2013. Is obesity a brain disease? Neurosci. Biobehav. Rev. 37, 2489-2503.

Smith, S.M., 2002. Fast robust automated brain extraction. Hum. Brain Mapp. 17, $143-155$.

Stiles, J., Jernigan, T.L., 2010. The Basics of Brain Development, pp. 327-348.

Takeuchi, H., Taki, Y., Hashizume, H., Asano, K., Asano, M., Sassa, Y., et al., 2015. The impact of television viewing on brain structures: cross-sectional and longitudinal analyses. Cerebr. Cortex 25, 1188-1197.

Tamnes, C.K., Roalf, D.R., Goddings, A.-L., Lebel, C., 2018. Diffusion MRI of white matter microstructure development in childhood and adolescence: methods, challenges and progress. Dev. Cogn. Neurosci. 33, 161-175.

Tellegen, P.J., Winkel, M., Wijnberg-Williams, B., Laros, J.A., 2005. Snijders-Oomen NietVerbale Intelligentietest: SON-R 2-1/2 -to-7. Ansterdam. Boom Testuitgevers.

Tomporowski, P.D., Davis, C.L., Miller, P.H., Naglieri, J.A., 2008. Exercise and children's intelligence, cognition, and academic achievement. Educ. Psychol. Rev. 20, 111-131.

Tremblay, M.S., Aubert, S., Barnes, J.D., Saunders, T.J., Carson, V., Latimer-Cheung, A.E., et al., 2017. Sedentary behavior research network (SBRN) - terminology consensus project process and outcome. Int. J. Behav. Nutr. Phys. Act. 14, 75.

Walsh, J.J., Barnes, J.D., Cameron, J.D., Goldfield, G.S., Chaput, J.-P., Gunnell, K.E., et al., 2018. Associations between 24 hour movement behaviours and global cognition in US children: a cross-sectional observational study. Lancet. Child Adolesc. Health 2, 783-791.

White, T., Muetzel, R.L., El Marroun, H., Blanken, L.M.E., Jansen, P., Bolhuis, K., et al., 2018. Paediatric population neuroimaging and the Generation R Study: the second wave. Eur. J. Epidemiol. 33, 99-125. 